\title{
An Outward-Wave-Favouring Finite Element-Based Strategy for Exterior Acoustical Problems
}

\author{
C. S. Jog \\ Facility for Research in Technical Acoustics (FRITA), Dept. of Mechanical Engg., Indian Institute of Science, \\ Bangalore 560012, India
}

(Received 30 November 2011, revised 10 September 2012, accepted 14 November 2012)

This work presents a finite element-based strategy for exterior acoustical problems based on an assumed pressure form that favours outgoing waves. The resulting governing equation, weak formulation, and finite element formulation are developed both for coupled and uncoupled problems. The developed elements are very similar to conventional elements in that they are based on the standard Galerkin variational formulation and use standard Lagrange interpolation functions and standard Gaussian quadrature. In addition and in contrast to wave envelope formulations and their extensions, the developed elements can be used in the immediate vicinity of the radiator/scatterer. The method is similar to the perfectly matched layer (PML) method in the sense that each layer of elements added around the radiator absorbs acoustical waves so that no boundary condition needs to be applied at the outermost boundary where the domain is truncated. By comparing against strategies such as the PML and wave-envelope methods, we show that the relative accuracy, both in the near and far-field results, is considerably higher.

\section{INTRODUCTION}

The main difficulty that arises in solving exterior acoustical problems is the unboundedness of the domain. Although the boundary element method yields accurate results, the computational cost and memory requirements for large-scale problems can be prohibitive. Finite element methods can be more costeffective due to the sparse nature of the matrices. Among the finite element-based techniques, one popular approach has been to truncate the computational domain at some distance away from the radiator or scatterer, and to impose a boundary condition at this artificial boundary (see Givoli ${ }^{1}$ for a description of the classical approaches and to Qi and Geers ${ }^{2}$ for a relatively recent approach known as the perfectly matched layer (PML)). Yet another approach involves infinite elements. ${ }^{3-9}$ Both approaches have their merits and demerits, which have been discussed in detail in the references cited.

Most of the work towards improving the former approach has focused either on devising and implementing higher-order absorbing conditions or on modifying these conditions to make them applicable for non-spherical truncation boundaries. The goal in this study is to improve upon the former approach, not by modifying the boundary conditions, but by modifying the function that is being solved for, namely the pressure field. The assumed form of the pressure field favours outgoing waves, which makes it easier for the resulting finite element formulation to capture the solution. Summarizing, note the following:

1. The proposed formulation is a modification of the conventional approach based on absorbing boundary conditions; the computational domain and input data are exactly the same; and the computational cost is also almost the same as for the conventional formulation.

2. The most crucial aspect of the proposed formulation is that it is based on a Cartesian framework and, thus, avoids the cumbersome use of angular coordinates and the problems associated with them. In particular, it allows the elements to be used directly in the vicinity of the radiator or scatterer, which is in contrast to existing wave envelope and related formulations, where an inner mesh of conventional elements has to be used, leading to poorer accuracy (see section 4.2 for a comparison). In addition, there is no problem of ill-conditioning, as for example, occurs in the radialdirection matrices in some infinite finite element formulations (see, e.g., Bhandakkar and $\mathrm{Jog}^{9}$ and Dreyer and Estorff ${ }^{10}$ ). Although researches have attempted to alleviate this ill-conditioning ${ }^{10}$ the fact remains that non-standard interpolations, such as Legendre or Jacobi polynomials, must be used.

3. Since an oscillatory part is separated out in the proposed formulation, the resulting finite element formulation has to capture a more gently varying function in most problems, resulting in much higher accuracy compared to the conventional formulation. In this connection, I mention that both the near- and far-field results presented in this study were obtained from raw nodal values without the use of the Helmholtz integral equation (which can be computationally very expensive to evaluate and which is used to obtain accurate far-field results ${ }^{3-5}$ ).

4. Since it uses only a first-order (spherical) damper, the implementation is very simple. Similar to the PML method, each layer of elements added around the radiator/scatterer absorbs acoustical waves so that no boundary condition needs to be applied at the truncated boundary. Note that the PML method is not only more complicated but also fails to yield good accuracy even on relatively simple problems, such as the dilatational motion of a spherical surface unless 